\title{
Rarest variations of ectopic pregnancy: an obstetrician's dilemma
}

\section{Kamlesh R. Chaudhari*, Ashwini Sakhalkar, Rajeshwari Khyade}

Department of Obstetrics and Gynecology, K. J. Somaiya Medical College and research centre, Mumbai, Maharashtra, India

Received: 31 March 2017

Accepted: 18 May 2017

\section{* Correspondence:}

Dr. Kamlesh R. Chaudhari,

E-mail: dr.kamleshchaudhari@gmail.com

Copyright: () the author(s), publisher and licensee Medip Academy. This is an open-access article distributed under the terms of the Creative Commons Attribution Non-Commercial License, which permits unrestricted non-commercial use, distribution, and reproduction in any medium, provided the original work is properly cited.

\begin{abstract}
Ectopic pregnancy is an important cause of morbidity and mortality which has always proven to be a challenge to obstetricians. Increased usage of artificial reproductive techniques has not only increased tubal ectopic pregnancies but also other rarer forms of ectopic pregnancies. With newer diagnostic and therapeutic tools, chance of early detection and intervention has increased many fold. Conservative treatment options have also gained importance. Here we discuss 3 peculiar types of rare ectopic pregnancies-heterotopic pregnancy, caesarean scar ectopic pregnancy and rudimentary horn ectopic pregnancy- and their treatment options.
\end{abstract}

Keywords: Artificial reproductive techniques, Caesarean scar pregnancy, Crown-rump length, Intrauterine insemination, Medical termination of pregnancy, Outpatient department

\section{INTRODUCTION}

An ectopic pregnancy, implantation of embryo at a site other than the uterine cavity, is most commonly seen in the fallopian tubes. However, due to the advent of newer assisted reproductive techniques, increasing incidences of caesarean delivery and better diagnostic tools such as ultrasonography and MRI, incidence of rarer forms of ectopic pregnancy and their early diagnosis is now possible. Also, because of newer surgical methods such as laparoscopy or interventional radiology, conservative management of these conditions and prevention of catastrophe can be done effectively. A high level of suspicion with a thorough knowledge of these rare forms of ectopic can significantly decrease morbidity and mortality. Some of these rare forms of ectopic pregnancy include-heterotopic pregnancy, caesarean scar pregnancy, rudimentary horn ectopic pregnancy. Heterotopic pregnancy, the presence of 2 gestational sacs simultaneously, is a rare event but with the advent of
ART is now an increasingly common complication. The reported incidence in spontaneous conception is 1 in $30,000 .{ }^{1}$ It is seen to have a multifactorial etiology. Tubal damage including scarring of tubal epithelium, damage of cilia or stenosis still remain the most important risk factors. $^{2}$ In assisted ART, the embryos that are placed in the endometrial cavity do not immediately implant onto the endometrium. They may drift towards the tubes and under the influence of corpus luteum, later, embed in the cavity. However, in the presence of an existing damaged tube, this journey may be interrupted, increasing chances of ectopic pregnancy and with higher order of embryos being placed in the womb predispose to heterotopic pregnancy. $^{2}$

Implantation of a pregnancy within the scar of a previous uterine caesarean scar is the rarest form of ectopic pregnancy. The incidence of CSP ranges from 1 in 1,800 to 1 in $2,060 .^{3}$ It is more common than previously thought. Patients with CSP have a risk of uterine rupture and life-threatening hemorrhage, which may lead to 
hysterectomy and may cause dramatic consequences for their future reproductive capacity. Because of the rarity of the condition, most of the CSPs in literature are case reports or small case series and thus therapeutic protocols have not been universally established. It is important that early and accurate diagnosis is obtained to avoid complications to preserve fertility. Several types of conservative treatments have been used-Dilatation and curettage, excision of the trophoblastic tissue by laparotomy or laparoscopy, local and or systemic administration of methotrexate, bilateral uterine artery embolization combined with curettage, bilateral internal iliac artery ligation with trophoblastic tissue evacuation and selective uterine artery embolization with curettage and or methotrexate administration. Pregnancy in a noncommunicating rudimentary horn is an extremely infrequent event that results from a transperitoneal migration of sperm or fertilized ovum. Incidence ranged from 1 in 76,000 to 1 in 1.5 lakh. ${ }^{4}$ When a rudimentary horn pregnancy is diagnosed, excision of the pregnant rudimentary horn is of crucial importance because 80 to $90 \%$ of these pregnancies eventually culminate in rupture. Less than $10 \%$ of these pregnancies occurring in the rudimentary horn reach term with fetal salvage rate between 0 to $13 \%$. $^{4}$

\section{Case 1}

35-year-old female Gravida 3 Abortion 2 with secondary infertility conceived after IUI with 10 weeks of amenorrhea presented with pain in abdomen and minimal spotting per vaginum. USG report was suggestive of live right tubal ruptured ectopic pregnancy with a CRL of $23 \mathrm{~mm}$ with presence of fetal pole and yolk sac and intrauterine missed abortion of 9 weeks with fetal pole seen within. There was hemoperitoneum of about $200 \mathrm{cc}$. Patient was hemodynamically stable. Emergency exploratory laparotomy with right salpingectomy with suction and evacuation of the missed abortion was performed. Histopathology report showed products of conception intrauterine with tubal ectopic pregnancy.

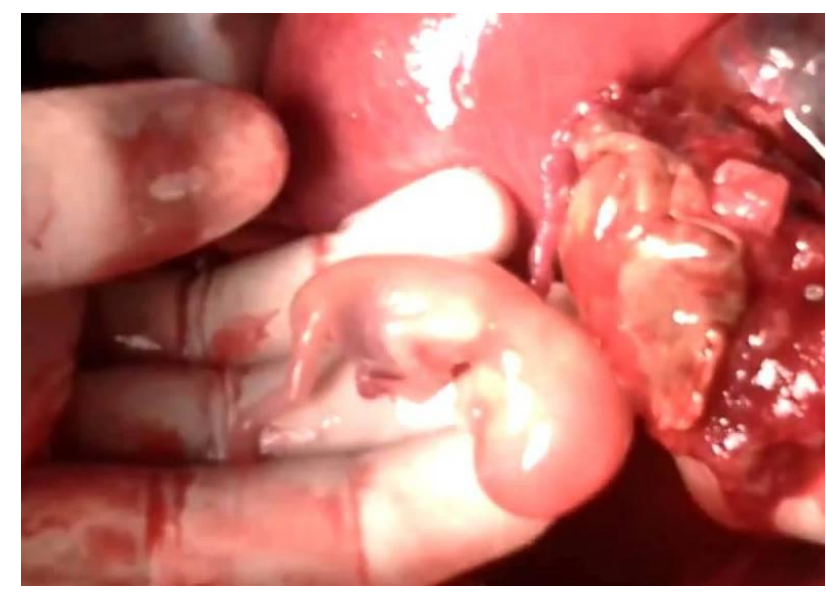

Figure 1: Intraoperative findings in ruptured tubal ectopic pregnancy.

\section{Case 2}

35-year-old female Gravida 2 Para 1 with previous history of caesarean section 5 years back with 2.5 months of amenorrhea was admitted at the tertiary care centre for bleeding per vaginum and pain in abdomen at 10.4 weeks of gestation.

Physical examination demonstrated stable vital signs while bimanual examination revealed an 8 weeks size uterus with no palpable adnexal mass. Transvaginal ultrasonography (TVS) showed a single live intrauterine pregnancy with a $36 \mathrm{~mm}$ gestational sac and a crownrump length (CRL) of $16 \mathrm{~mm}$ with the sac located in the lower anterior wall of the uterus with a myometrial thickness of $3 \mathrm{~mm}$ between the gestational sac and the bladder. The gestational sac was surrounded by a rich blood flow signal. These signs were suggestive of a live scar ectopic pregnancy.

Patient was counselled regarding the management options. As she had a caesarean delivery in the past, possibility of uterine rupture was explained due to presence of a live gestation. As patient wanted to preserve her reproductive capacity and did not prefer a long hospital stay, decision to proceed with curettage and aspiration guided by laparoscopy was taken. In the laparoscopic approach, we detached the uterovesical fold of peritoneum. Suction and curettage of the scar ectopic pregnancy was done under laparoscopic guidance followed by suturing of the rent at scar site using barbed sutures. Patient recovered uneventfully. Scar tissue sent for histopathology, confirmed the diagnosis.

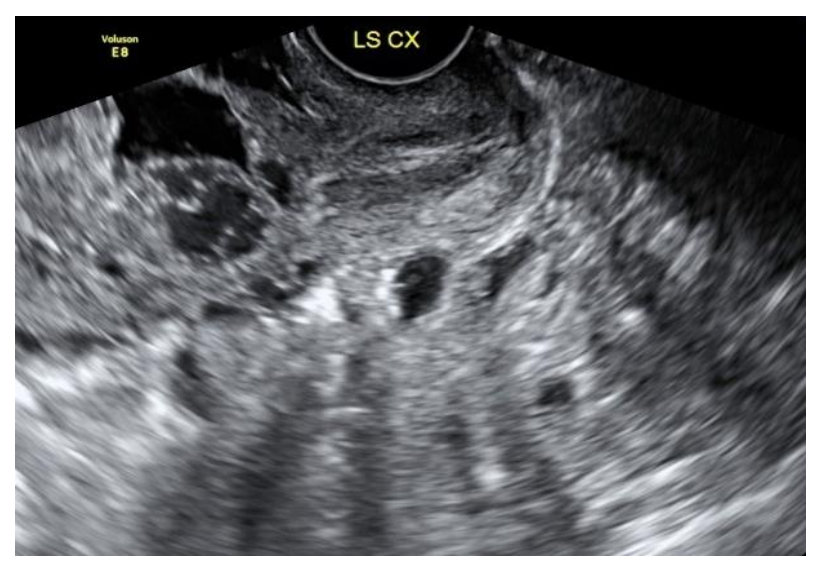

Figure 2: Ultrasound picture of scar ectopic pregnancy.

Case 3

28-year-old female Gravida 2 Para 1 with 2 months of amenorrhea came to the tertiary care centre OPD with an ultrasonography report suggestive of left tubal ectopic pregnancy. She gave history of taking MTP pills from a local physician with no bleeding following it after which a USG was done. On examination, patient was 
hemodynamically stable with bimanual examination revealing a bulky uterus with no palpable adnexal mass. Cervical motion tenderness could not be elicited. TVS showed left adnexa having a sac with a thick echogenic mass measuring $3.1 \mathrm{~cm} \mathrm{X} 3 \mathrm{~cm}$ with a sac measuring 1.7 $\mathrm{cm}$ corresponding to 6.1 weeks of gestation with presence of fetal pole and yolk sac within. Serum beta-HCG done was $7700 \mathrm{IU} / \mathrm{ml}$. Decision to proceed with exploratory laparotomy for unruptured tubal ectopic pregnancy was taken.

Intraoperatively, there was presence of a noncommunicating rudimentary horn on the left side of the non-gravid uterus with the round ligament and fallopian tube attached to its left side. Both fallopian tubes and ovaries were found to be normal with corpus luteal cyst seen in the right ovary. As patient wanted to conserve her reproductive capacity, an incision was taken on the antimesenteric border of the rudimentary horn and the products of conception were evacuated followed by left salpingectomy.

Postoperative period was uneventful. Histopathology report of the evacuated products confirmed the diagnosis of pregnancy. Serial beta-HCG levels showed a falling trend and came to non-pregnant level after 6 weeks.

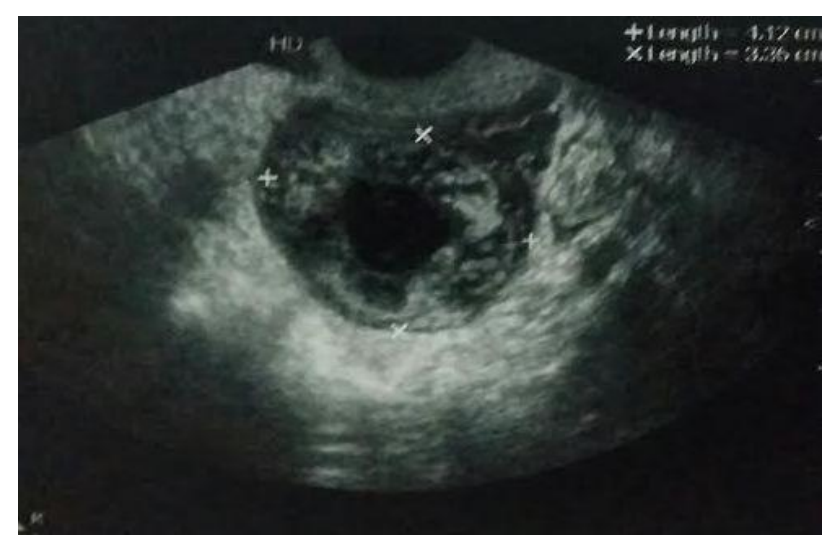

Figure 3: Ultrasound finding of rudimentary horn ectopic pregnancy.

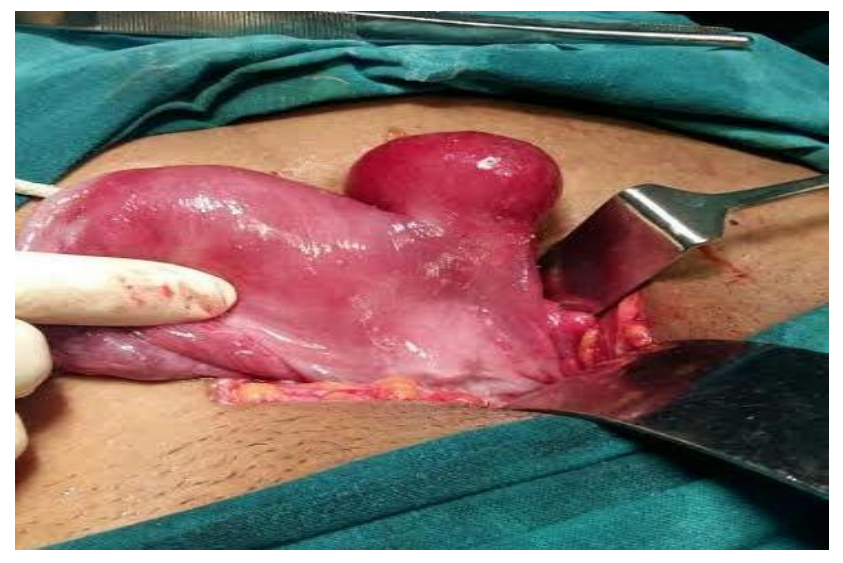

Figure 4: Intraoperative picture of exploration for rudimentary horn ectopic pregnancy.

\section{DISCUSSION}

A heterotopic gestation is difficult to diagnose clinically and is not as straightforward as the diagnosis of ectopic pregnancy.

Heterotopic pregnancy often goes unnoticed because of presence of an intrauterine gestational sac. These patients usually present with lower abdominal pain in $1^{\text {st }}$ trimester. USG examination especially TVS has proven to be an invaluable tool in the diagnosis of this condition. The extrauterine pregnancy in cases of heterotopic pregnancy has a similar sonographic appearance as that of an isolated ectopic pregnancy.

With the growing number of caesarean deliveries CSP is becoming a more common phenomenon than was earlier thought. TVS is the preferred diagnostic method for CSP. The USG criteria to diagnose CSP the gestational sac being partly in the anterior part of the uterine isthmus leading to discontinuity of the anterior wall of the uterus. ${ }^{5}$ In addition, the myometrial layer between the urinary bladder and the gestational sac is thin and attenuated and usually less than $0.5 \mathrm{~cm}$. The presence of blood flow signal around the gestational sac as seen on Doppler ultrasonography helps the diagnosis.

In this case, the diagnosis was based on a previous history of caesarean scar and USG criteria confirming it. As compared to other management options for CSP such as conservative management, blind uterine curettage and aspiration, the greatest advantage of curettage and aspiration guided by laparoscopy was that, this method seemed to decrease the risk of uterine rupture and failure of treatment of CSP. ${ }^{5}$ The estimated blood loss was less than $50 \mathrm{cc}$ which is less than other management options. Problems with methotrexate therapy like need for several cycles and side-effects such as leukopenia, stomatitis, alopecia, renal and hepatic dysfunction or with bilateral uterine artery embolization like reduced ovarian function or decreased uterine blood flow is not seen in the management applied in this case making it a preferred method of treatment.

Curettage and aspiration guided by laparoscopy is a promising method of treatment of CSP especially for those who wish to have a shorter hospital stay. Rudimentary horn with a unicornuate uterus results from failure of complete development of one of the mullerian ducts and incomplete fusion with the contralateral side. In $83 \%$ of cases the rudimentary horn is noncommunicating. ${ }^{6}$

Earlier most rudimentary horn ectopics presented in ruptured state. However due to better diagnostic tool like ultrasonography, MRI, pre-rupture diagnosis is possible. The treatment of choice is a complete excision of the rudimentary horn from its base followed by suturing of the lateral rent on the wall of the uterus. This may be risky for future pregnancies as a weakened lateral uterine 
wall may cause spontaneous uterine rupture in the $3^{\text {rd }}$ trimester. The method of treatment applied here ensured no breach in the uterine wall making it the treatment of choice in unruptured non-communicating rudimentary horn ectopic pregnancy.

\section{CONCLUSION}

These rarest forms of ectopic pregnancy have gained importance in modern obstetrics with increased usage of artificial reproductive techniques. They have presentations much more varied than the classical tubal ectopic pregnancy making their detailed study of utmost importance. History of infertility and artificial reproductive techniques should raise high suspicion. In cases with high risk for ectopic pregnancies, early USG is advisable with serum beta-HCG and or MRI if found necessary. Intervention should be proceeded with after a detailed history and examination supported by definitive radiological diagnosis and conservative methods of treatment should be taken into consideration. Basically, one correctly, early, promptly treated obstetric emergency is one mother and hence one family saved!!!

Funding: No funding sources Conflict of interest: None declared Ethical approval: Not required

\section{REFERENCES}

1. Noor N, Bano I, Parveen S. Heterotopic pregnancy with successful pregnancy outcome. J Hum Reprod Sci. 2012;5(2):213-4.

2. Deka D, Bahadur A, Singh A, Malhotra N. Successful management of heterotopic pregnancy after fetal reduction using potassium chloride and methotrexate. J Hum Reprod Sci. 2012;5(1):57-60.

3. Collins K, Kothari A. Catastrophic consequences of a caesarean scar pregnancy missed on ultrasound. Australasian J Ultrasound in Med. 2015;18(4):150-6.

4. Sönmezer M, Taskin S, Atabekogğlu C, Güngör M, Ünlü C. Laparoscopic Management of Rudimentary Uterine Horn Pregnancy: Case Report and Literature Review. J Soc Laparoendoscopic Surg. 2006;10(3):396-399.

5. Shu S, Luo X, Wang Z, Yao Y. Cesarean scar pregnancy treated by curettage and aspiration guided by laparoscopy. Therapeutics and Clinical Risk Management. 2015;11:1139-41.

6. Ambusaidi Q, Jha C. Pregnancy in the Rudimentary Uterine Horn: Case report of an unusual presentation. Sultan Qaboos Uni Med J. 2014;14(1):e134-e138.

Cite this article as: Chaudhari KR, Sakhalkar A, Khyade R. Rarest variations of ectopic pregnancy: an obstetrician's dilemma. Int J Reprod Contracept Obstet Gynecol 2017;6:3173-6. 